\title{
Forming competing fear learning and extinction memories in adolescence makes fear difficult to inhibit
}

\author{
Kathryn D. Baker and Rick Richardson \\ School of Psychology, UNSW Australia, Sydney, New South Wales 2052, Australia
}

\begin{abstract}
Fear inhibition is markedly impaired in adolescent rodents and humans. The present experiments investigated whether this impairment is critically determined by the animal's age at the time of fear learning or their age at fear extinction. Male rats ( $n=170$ ) were tested for extinction retention after conditioning and extinction at different ages. We examined neural correlates of impaired extinction retention by detection of phosphorylated mitogen-activated protein kinase immunoreactivity (pMAPK-IR) in several brain regions. Unexpectedly, adolescent rats exhibited good extinction retention if fear was acquired before adolescence. Further, fear acquired in adolescence could be successfully extinguished in adulthood but not within adolescence. Adolescent rats did not show extinction-induced increases in pMAPK-IR in the medial prefrontal cortex or the basolateral amygdala, or a pattern of reduced caudal central amygdala pMAPK-IR, as was observed in juveniles. This dampened prefrontal and basolateral amygdala MAPK activation following extinction in adolescence occurred even when there was no impairment in extinction retention. In contrast, only adolescent animals that exhibited impaired extinction retention showed elevated pMAPK-IR in the posterior paraventricular thalamus. These data suggest that neither the animal's age at the time of fear acquisition or extinction determines whether impaired extinction retention is exhibited. Rather, it appears that forming competing fear conditioning and extinction memories in adolescence renders this a vulnerable developmental period in which fear is difficult to inhibit. Furthermore, even under conditions that promote good extinction, the neural correlates of extinction in adolescence are different than those recruited in animals of other ages.
\end{abstract}

[Supplemental material is available for this article.]

Adolescence is often described as a developmental window of vulnerability, and is a period in which anxiety disorders commonly emerge (Kim-Cohen et al. 2003). This developmental period is a critical time for interventions because adolescent fear disorders are a strong predictor for adult anxiety and other psychological disorders (Kessler et al. 2012). Although extinction of learned fear is widely accepted as a valid model for exposure-based therapy (Graham and Milad 2011), there is a relatively small body of research on fear extinction in adolescent rodents and humans (Baker et al. 2014; Casey et al. 2015). A prominent finding of these studies is that retention of fear extinction is impaired in this stage of development, relative to both younger and older age groups (McCallum et al. 2010; Kim et al. 2011; Pattwell et al. 2012). This impaired fear inhibition could be caused by inefficient recruitment of the medial prefrontal cortex (mPFC) during extinction in adolescents. In adult and juvenile animals, the mPFC is critical for the consolidation and retrieval of extinction. Some studies have shown that the infralimbic (IL) subdivision of the mPFC, in contrast to the prelimbic (PL) subdivision, is particularly important for extinction (Morgan et al. 1993; Milad and Quirk 2002; Sierra-Mercado et al. 2006; Kim et al. 2009) while other studies have reported similar results for the IL and PL (Santini et al. 2004; Kim et al. 2010; Park and Choi 2010). In any case, NMDA receptors in the $\mathrm{MPFC}$, and their downstream signaling cascades (including mitogen-activated protein kinases [MAPK]), are essential for the consolidation of extinction (Burgos-Robles et al. 2007). The phosphorylation of MAPK (pMAPK) is involved in the activity-dependent modulation of synaptic plasticity in various limbic brain regions (English and Sweatt 1997; Huang et al. 2000). However, adolescent rodents do not exhibit the same

\section{Corresponding author: k.baker@unsw.edu.au}

Article is online at http://www.learnmem.org/cgi/doi/10.1101/lm.039487. 114. extinction-induced increases in pMAPK, a marker of neuronal activity (c-Fos expression), or synaptic plasticity (measured by electrophysiological recordings) in the IL that are observed in juveniles and adults (Kim et al. 2011; Pattwell et al. 2012). This lack of neuronal synaptic plasticity in the mPFC after extinction is consistent with the impaired extinction retention exhibited by adolescents. Nevertheless, it is unknown whether other regions involved in the expression, retrieval, and/or extinction of learned fear, in particular the amygdala (Herry et al. 2006; Sotres-Bayon et al. 2007) and paraventricular thalamus (PVT) (Padilla-Coreano et al. 2012; Li et al. 2014; Do-Monte et al. 2015), show activity-dependent changes in response to fear extinction in adolescence.

Impaired extinction retention in adolescents is a robust, reliable finding that has been replicated across species (i.e., mice, rats, and humans) (McCallum et al. 2010; Kim et al. 2011; Pattwell et al. 2012). It has generally been assumed that adolescents show impaired fear inhibition because of their age at extinction. However, in all of the studies to date that have demonstrated impaired extinction retention in adolescence, groups differed not only in their age at the time of extinction but also their age at the time of fear learning. That is, the participants, whether human or rodent, acquired the fear when juveniles, adolescents, or adults as well as having the fear extinguished at these ages. The present experiments investigated whether it is the animals' age at the time of fear learning or their age at fear extinction that determines if extinction is impaired in adolescence. It was hypothesized that

(C) 2015 Baker and Richardson This article is distributed exclusively by Cold Spring Harbor Laboratory Press for the first 12 months after the full-issue publication date (see http://learnmem.cshlp.org/site/misc/terms.xhtml). After 12 months, it is available under a Creative Commons License (AttributionNonCommercial 4.0 International), as described at http://creativecommons. org/licenses/by-nc/4.0/. 
extinction retention would be impaired in adolescents regardless of their age when fear was learned. In addition, we further characterized the neural mechanisms mediating the impaired extinction in adolescents by not only examining the activity-dependent expression of the neuronal synaptic plasticity marker pMAPK (Sweatt 2004) in the mPFC (to replicate past results) but also in the amygdala and PVT.

\section{Results}

Experiment 1: do animals extinguished in adolescence have impaired fear extinction regardless of their age at fear acquisition?

Experiment 1 tested the prediction that fear memories extinguished in adolescence, regardless of whether the fear was acquired before or during adolescence, would be vulnerable to relapse. There were three groups. In two groups, juvenile (Postnatal day [P]24) and adolescent (P34-35) animals received Pavlovian fear conditioning, extinction, and test on consecutive days (Groups JuvCond-Ext and AdolesCond-Ext, respectively). The group of particular interest was the third group, which received fear conditioning as juveniles (P24) and after $\sim 10 \mathrm{~d}$ received extinction and test as adolescents (Group JuvCond-AdolesExt). We predicted that because of their age at the time of extinction, these animals would show impaired extinction retention.

Conditioning and extinction proceeded as expected in all experiments. In each experiment, all groups showed comparable overall levels of fear and rates of fear acquisition and extinction (Supplemental Fig. S1-5).
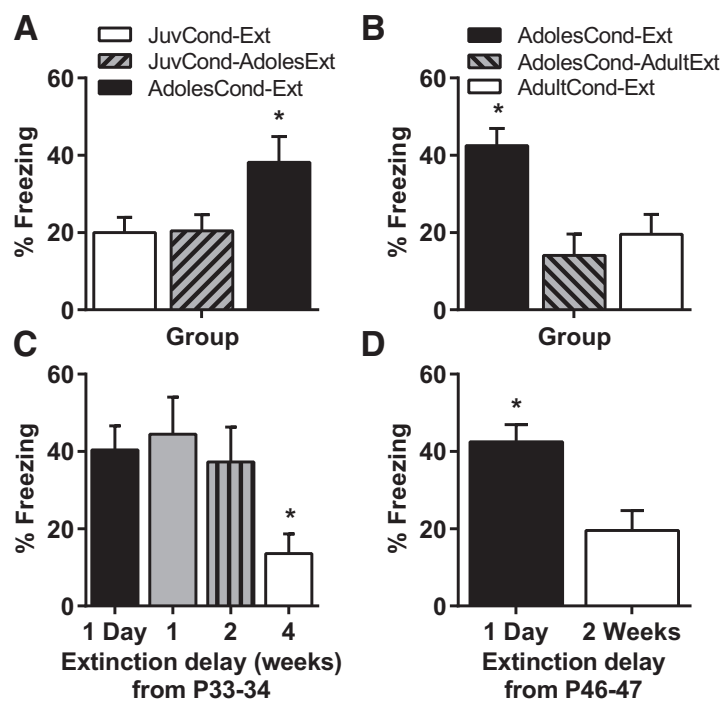

Figure 1. Impaired retention of fear extinction in adolescence is only observed when conditioning and extinction both occur in adolescence. (A) Mean ( \pm SEM) levels of CS-elicited freezing during test in Experiment 1. Rats were in one of three groups: JuvCond-Ext $(n=12)$, JuvCondAdolesExt $(n=11)$, or AdolesCond-Ext $(n=11)$. (B) Mean $( \pm$ SEM) levels of CS-elicited freezing during test in Experiment 2. Rats were in one of three groups: AdolesCond-Ext $(n=12)$, AdolesCond-AdultExt $(n=11)$, or AdultCond-Ext $(n=11)$. (C) Mean $( \pm$ SEM) levels of CSelicited freezing during test in Experiment 3. Rats were in one of four groups where extinction occurred either $1 \mathrm{~d}(n=12), 1 \mathrm{wk}(n=9), 2$ wk $(n=10)$, or $4 \mathrm{wk}(n=12)$ after conditioning in adolescence at P33-34. (D) Mean ( \pm SEM) levels of CS-elicited freezing during test in Experiment 4 . Rats were in one of two groups where extinction occurred either $1 \mathrm{~d}(n=12)$ or $2 \mathrm{wk}(n=12)$ after conditioning in late adolescence at P46-47. (*) Indicates significant difference compared with all the other groups $(P<0.05)$.
In Experiment 1, there were group differences at test $\left(F_{(2,31)}=\right.$ 4.22, $P=0.024)$. Figure $1 \mathrm{~A}$ shows that we replicated the finding that AdolesCond-Ext rats exhibited impaired extinction retention. Surprisingly, adolescent rats exhibited good extinction retention if fear was acquired before adolescence (Group JuvCondAdolesExt). Student-Newman-Keuls post hoc tests confirmed that the JuvCond-Ext and JuvCond-AdolesExt animals showed similar levels freezing at test and these differed significantly from AdolesCond-Ext animals $(P<0.05)$. In contrast to our predictions, these findings demonstrate fear acquired prior to adolescence can be successfully extinguished during adolescence. Therefore, the animal's age at extinction does not determine whether extinguished fear is vulnerable to relapse.

\section{Experiment 2: do animals trained as adolescents have impaired fear extinction regardless of their age at extinction?}

We next tested whether the animal's age at fear learning determines whether impaired extinction retention is exhibited by examining whether animals conditioned during adolescence show impaired extinction retention even if extinction occurs in adulthood. There were three groups. Two groups (one adolescent and one adult) received fear conditioning, extinction, and test on consecutive days (Groups AdolesCond-Ext and AdultCond-Ext, respectively). The third group received fear conditioning as adolescents and after $\sim 5 \mathrm{wk}$, extinction and test as adults (Group AdolesCond-AdultExt). Animals in the three groups were either P34-37 or $\sim$ P70 at extinction.

There were group differences at test $\left(F_{(2,31)}=9.22, P=0.001\right.$; Fig. 1B). Both the AdolesCond-AdultExt and AdultCond-Ext groups showed similar low levels of freezing at test, and these two groups differed from the AdolesCond-Ext animals $(P<$ 0.05). In summary, rats that were fear conditioned in adolescence but extinguished in adulthood, exhibited good extinction retention compared with animals trained and extinguished in adolescence. Thus, the animals' age at the time of fear learning does not determine whether fear extinction retention is impaired.

\section{Experiment 3: delaying extinction in adolescence does not improve extinction retention}

The findings from the first two Experiments suggest that it is neither the animals' age at the time of fear acquisition nor their age at fear extinction that determines whether deficits in extinction retention are observed. Rather, it appears that impaired extinction retention in adolescence requires that both fear conditioning and extinction occur in adolescence. These results were surprising so we next examined whether delaying extinction in adolescence improves extinction, in order to test whether both recent and older (i.e., remote) fear memories are resistant to extinction in adolescence. Adolescent rats received extinction either $1 \mathrm{~d}$ (at P34-35), 1 wk (at P40-41), or 2 wk (at P47-48) after conditioning (all still within adolescence). A fourth group received conditioning as adolescents and extinction $4 \mathrm{wk}$ later as young adults (at P61-62).

There were group differences at test $\left(F_{(3,39)}=3.77, P=\right.$ 0.018 ). Animals in groups which received extinction $1 \mathrm{~d}, 1$ or 2 wk after extinction showed similar levels of freezing at test (Fig. 1C), and these were significantly higher compared with animals that received extinction in early adulthood $4 \mathrm{wk}$ after conditioning $(P<0.05)$. That is, extinction retention was impaired in rats conditioned as adolescents even if extinction occurred $2 \mathrm{wk}$ after conditioning (but still within adolescence). Extinction retention only improved once extinction was delayed until when animals reached adulthood. 


\section{Experiment 4: Extinction retention is impaired in late adolescence}

Experiment 4 tested the possibility that the improvement in extinction retention observed in adult animals which learned fear as adolescents in Experiments 2 and 3 was due to the age of the animals rather than the longer interval between conditioning and extinction. It is possible that the strength of the fear memory could have decayed over time and led to improved fear extinction at the later time. Although the similar levels of fear and rates of extinction in Experiments 2 and 3 (Supplemental Figs. S2, S3) do not support this explanation, we tested these competing accounts in this experiment. All rats received fear conditioning in late adolescence (P46-47). Extinction and test occurred either the following day (P47-48) or $2 \mathrm{wk}$ after conditioning (P61-62) as adults.

There were group differences at test (Fig. 1D). Late adolescent animals receiving extinction the day after conditioning showed impaired extinction retention compared with those that received extinction 2 wk after conditioning $\left(t_{(22)}=2.50, P=0.02\right)$. Given that in Experiment 3 extinction retention was impaired in rats conditioned as adolescents if extinction occurred $2 \mathrm{wk}$ after conditioning, but in this experiment fear acquired in late adolescence could be successfully extinguished 2 wk later in adulthood, suggests that the improvement in extinction retention observed in adult animals in Experiments 2 and 3 was due to the age of the animals rather than the longer interval between conditioning and extinction.

\section{Experiment 5: Neural markers of impaired extinction in adolescent rats}

In order to examine the neural mechanisms underlying impaired extinction retention in adolescent rats, we replicated the design of Experiment 1, having the same three groups (i.e., JuvCond-Ext, JuvCond-AdolesExt, and AdolesCond-Ext). A "No Extinction" control group was added that received conditioning and was then exposed to the extinction context without any CS presentations. This group consisted of four animals from each condition collapsed into a single nonextinguished control $(n=12)$ after analyses determined that there were no group differences for freezing or pMAPK-immunoreactive (IR) neuron counts (see Supplemental Results). Using a second series of brain sections, we compared pMAPK-IR neuron counts in subregions of the mPFC, amygdala, and PVT in a randomly chosen subset of the No Extinction animals $(n=7)$ to experimentally naive (home cage) animals $(n=7)$. There were no significant group differences in pMAPK expression in any of these brain regions between the Naive and No Extinction animals (Supplemental Fig. S6A-C). These results are similar to those observed in the basolateral amygdala (BLA) in adult rats (Herry et al. 2006) and confirm that the No Extinction group was an appropriate control group to assess extinction-induced changes in pMAPK.
We measured pMAPK following extinction as it is involved in the activity-dependent modulation of synaptic plasticity in various limbic brain regions (English and Sweatt 1997; Huang et al. 2000; Sweatt 2004). One hour following the extinction/exposure session, rats were perfused and the phosphorylation of MAPK in the MPFC, amygdala, PVT, and lateral habenula was assessed by immunohistochemistry. This time-point for sacrifice after extinction was selected based on previous reports that pMAPK-IR neuron counts peak $1 \mathrm{~h}$ after extinction in limbic regions (e.g., BLA and hippocampus; Herry et al. 2006; Fischer et al. 2007). Figures 2 and 3 depict the number of pMAPK-IR neurons observed in the PL and IL cortices of the MPFC, and the BLA and central amygdala (CeA), respectively. We predicted that the two groups that exhibited good extinction retention in Experiment 1 (i.e., JuvCond-Ext and JuvCond-AdolesExt) would show an extinction-induced increase in PMAPK-IR in the IL and BLA, and a decrease in pMAPK-IR in the CeA, and these changes would not be observed in the AdolesCond-Ext animals that exhibited impaired extinction retention.

In the $\mathrm{mPFC}$, there were group differences in pMAPK-IR neuron counts for both the PL $\left(F_{(3,26)}=3.23, P=0.039\right)$ and IL $\left(F_{(3,26)}=4.04, P=0.012\right)$ (Fig. $\left.2 \mathrm{~A}-\mathrm{C}\right)$. In contrast to our predictions, Dunnett's post hoc tests showed that only JuvCond-Ext animals showed an extinction-induced increase in pMAPK-IR in the PL $(P=0.017)$ and IL $(P=0.004)$, whereas animals which received extinction in adolescence, regardless of their age at the time of fear acquisition (i.e., JuvCond-AdolesExt or AdolesCondExt) did not $(P>0.05)$. In the amygdala, there were group differences in pMAPK-IR neuron counts in BLA $\left(F_{(3,26)}=8.48, P<\right.$ $0.001)$ and a trend toward a group difference in the CeA $\left(F_{(3,26)}=2.70, P=0.067\right.$ ) (Fig. 3A,B). In a similar pattern to the mPFC, JuvCond-Ext animals showed a significant extinctioninduced increase in pMAPK-IR in the BLA (Dunnett's $P=0.001$; both rostral and caudal regions, smallest $F_{(3,26)}=7.53, P<$ 0.001 , Dunnett's $P=0.002$; Supplemental Fig S7A) but this was not observed in animals which received extinction in adolescence, regardless of their age at the time of fear acquisition $(P>$ $0.05)$. There were group differences in pMAPK-IR neuron counts in the caudal $\left(F_{(3,26)}=3.15, P=0.042\right)$ but not rostral $\left(F_{(3,26)}=\right.$ $1.61, P>0.05)$ CeA. Post hoc tests showed that only JuvCondExt animals showed a trend toward reduced activation of the caudal CeA with extinction $(P=0.057$; Supplemental Fig. S7A). The ratio of BLA to CeA pMAPK-IR within animals also differed across conditions $\left(F_{(3,26)}=11.36, P<0.001\right.$; Fig. 3C). Adolescent animals (i.e., JuvCond-AdolesExt and AdolesCond-Ext), showed a pattern of low BLA pMAPK-IR and high CeA pMAPK-IR after extinction that did not differ significantly from that in No Extinction animals $(P>0.05)$. Importantly, the BLA/CeA ratio was higher after extinction in JuvCond-Ext animals compared with No Extinction animals $(P<0.001)$, with increased BLA and reduced CeA pMAPK expression. Taken together, these results
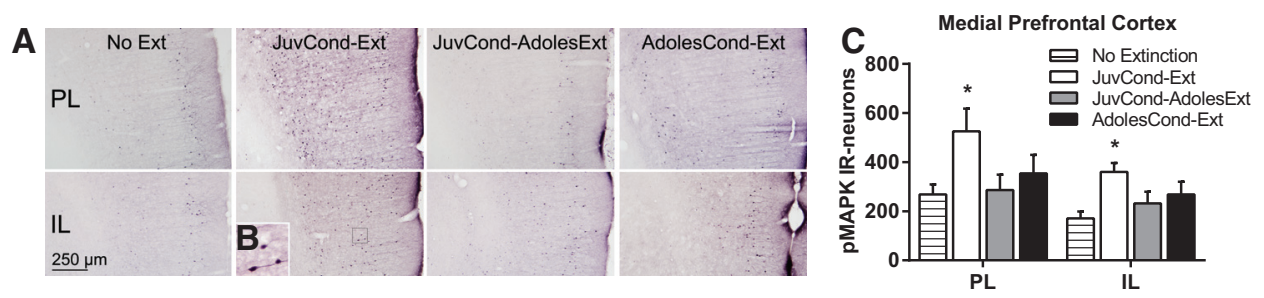

Figure 2. Prefrontal cortex PMAPK expression following extinction in adolescence. (A) Representative photomicrographs of pMAPK-immunoreactivity (pMAPK-IR) in the prelimbic cortex (PL) and infralimbic cortex (IL). (B) Example pMAPK-IR neurons in the IL. (C) Mean ( \pm SEM) counts of pMAPK-IR in the PL and IL subregions of the medial prefrontal cortex. Rats were in one of four groups: No Extinction $(n=12)$, JuvCond-Ext $(n=6)$, JuvCond-AdolesExt $(n=6)$, or AdolesCond-Ext $(n=6)$. $\left({ }^{*}\right)$ Indicates significant difference compared with No Extinction controls $(P<0.05)$. 
A

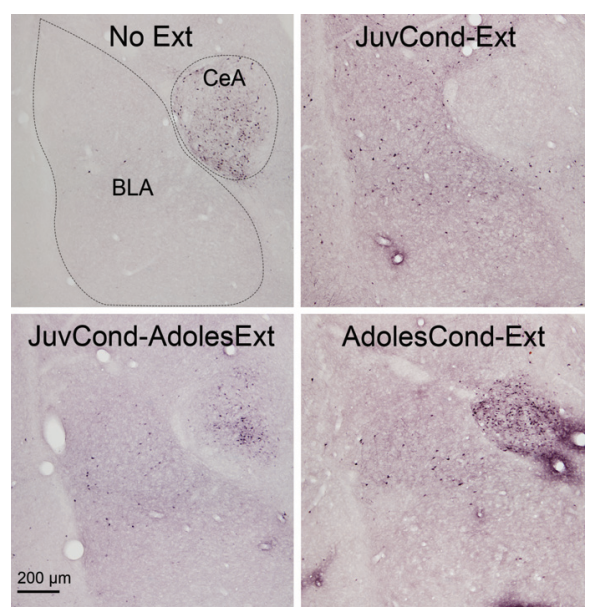

B
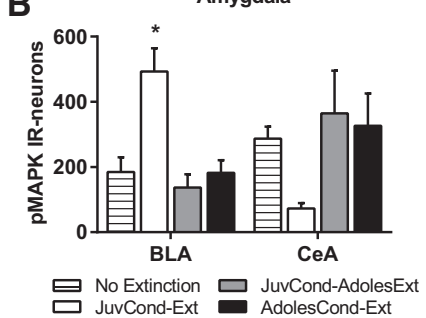

C

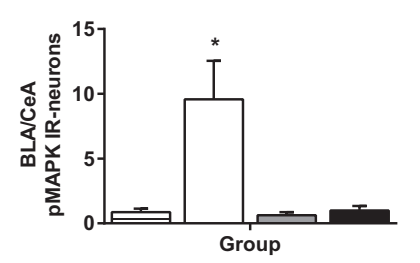

Figure 3. Amygdala pMAPK expression following extinction in adolescence. $(A)$ Representative photomicrographs of pMAPK-IR in the caudal amygdala showing the basolateral amygdala (BLA) and central amygdala $(\mathrm{CeA})$. (B) Mean ( \pm SEM) counts of pMAPK-IR in the amygdala. (C) Ratio of pMAPK-IR in the BLA to CeA amygdala. Rats were in one of four groups: No Extinction $(n=12)$, JuvCond-Ext $(n=6)$, JuvCond-AdolesExt $(n=6)$, or AdolesCond-Ext $(n=6)$. (*) Indicates significant difference compared with No Extinction controls $(P<0.05)$.

suggest that in both the mPFC and the amygdala, expression of pMAPK, a marker of synaptic plasticity, was determined more by the animal's age rather than whether they exhibited good extinction retention or not (in Experiment 1).

Because the dampened prefrontal and BLA pMAPK expression following extinction in adolescence was observed even when there was no impairment in extinction retention, we examined activation of MAPK in the PVT, a region implicated in the retrieval and/or expression of fear memories (Do-Monte et al. 2015). As this region is not usually involved in extinction (Padilla-Coreano et al. 2012; Li et al. 2014), we predicted that the JuvCond-Ext and JuvCond-AdolesExt groups would not show extinction-induced increases in the PVT, but that the AdolesCond-Ext group may show higher activation of MAPK in this region. We also examined the lateral habenula given its involvement in learned fear and processing of stimuli predicting punishment (Ressler et al. 2002; Matsumoto and Hikosaka 2009) but did not observe any group differences (Supplemental Fig. S7B).

Figure 4 depicts the number of pMAPK-IR neurons observed in the anterior, middle, and posterior PVT. Across conditions, a different pattern than that observed in the mPFC and amygdala occurred in the PVT, in that animals showed different activation of MAPK in this region reflecting their extinction retention perfor-

olescence. These findings demonstrate that neither the animal's age at the time of fear learning or extinction determines whether fear extinction is impaired. Rather, it appears that the impairment in extinction retention previously reported in adolescence (McCallum et al. 2010; Kim et al. 2011; Pattwell et al. 2012; Baker et al. 2013) requires both fear conditioning and extinction occur in adolescence (Fig. 5).

Successful learning and retention of fear extinction require functional coupling between the IL and BLA, in both juveniles and adults (Milad and Quirk 2002; Herry et al. 2008; Kim et al. 2009; Knapska et al. 2012). We examined the neural correlates of impaired extinction retention in adolescence by measuring the expression of the neuronal synaptic plasticity marker pMAPK. The prediction was that the two groups that exhibited good extinction retention in Experiment 1 (i.e., JuvCond-Ext and JuvCond-AdolesExt) would show activation of MAPK in the IL and BLA during extinction and this would not be observed in the AdolesCond-Ext group that exhibited impaired extinction retention. In JuvCond-Ext animals, we replicated previous findings of an extinction-induced increase in pMAPK expression in the BLA (Kim et al. 2009) and IL (Kim et al. 2009, 2011). In contrast to that earlier work, we also observed increased pMAPK-IR in the PL following extinction in rats conditioned and extinguished as juveniles. In a previous study, significantly higher mPFC pMAPK expression in both the PL and IL following extinction training
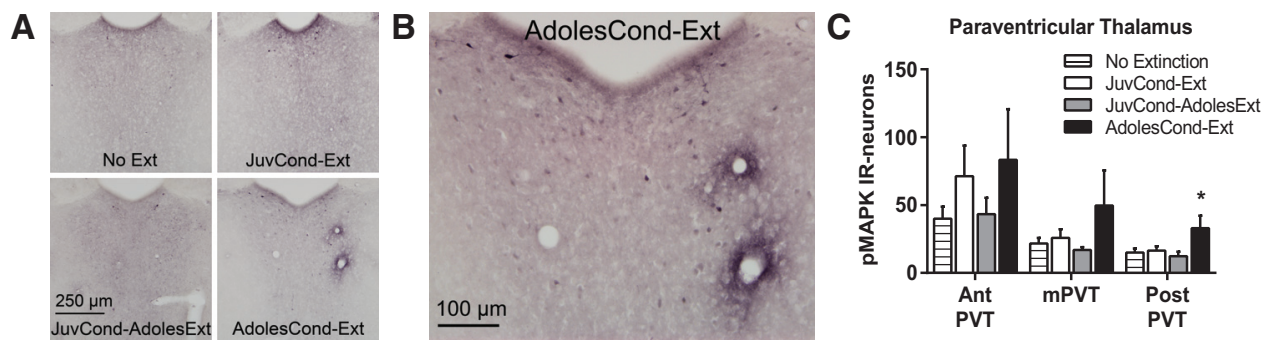

Figure 4. Paraventricular thalamus (PVT) pMAPK expression following extinction in adolescence. $(A, B)$ Representative photomicrographs of pMAPK-IR in the posterior PVT. (C) Mean ( \pm SEM) counts of pMAPK-IR in the anterior (AntPVT), middle (mPVT), and posterior (PostPVT) PVT. Rats were in one of four groups: No Extinction $(n=12)$, JuvCond-Ext $(n=6)$, JuvCond-AdolesExt $(n=6)$, or AdolesCond-Ext $(n=6)$. $\left({ }^{*}\right)$ Indicates significant difference compared with No Extinction controls $(P<0.05)$. 


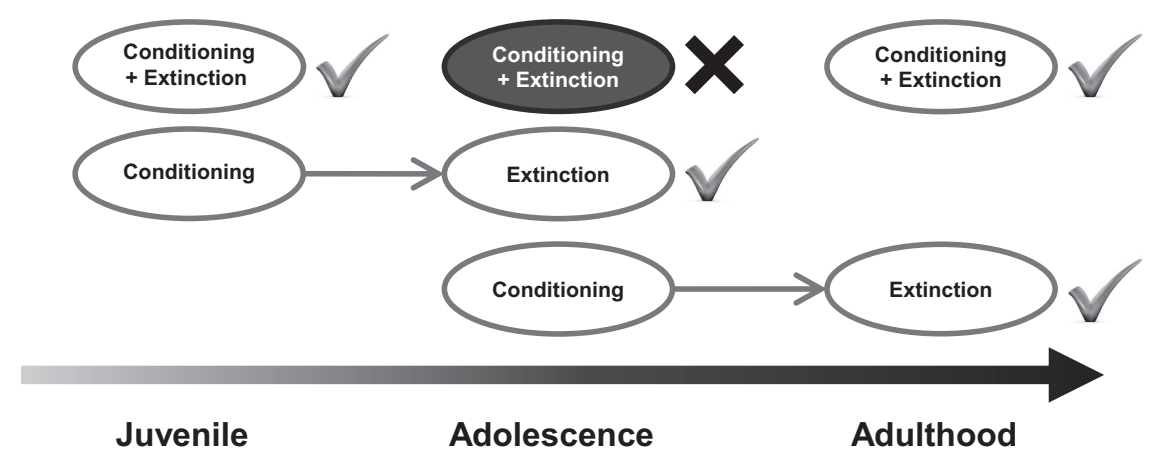

Figure 5. A schematic of extinction retention across development. Neither the animal's age at the time of fear learning or at the time of extinction determines whether fear extinction is impaired. Rather, it appears that the impairment in extinction retention in adolescence requires both fear conditioning and extinction occur in adolescence.

was observed in adolescents given double the number of extinction trials (Kim et al. 2011). There are also several studies which report increased neural activity measured by c-Fos expression in both the PL and IL after extinction training in adult animals (Santini et al. 2004; Kim et al. 2010; Park and Choi 2010). Further studies are needed to reconcile such findings with the view that the PL and IL exert opposing effects on fear expression and inhibition (Sotres-Bayon and Quirk 2010). In our study increased pMAPK expression in the MPFC and BLA in the JuvCond-Ext animals was associated with reduced pMAPK in the caudal CeA, consistent with a suppression of fear responses (Orsini and Maren 2012).

AdolesCond-Ext animals did not show increased IL or PL pMAPK expression in response to extinction, replicating previous reports (Kim et al. 2011), but they also showed reduced activation of MAPK in the BLA. This latter finding is the first (to our knowledge) that adolescence appears to be a unique developmental period in which extinction does not increase neuronal synaptic plasticity in the BLA (indexed by pMAPK-IR), an effect that is observed not only in juveniles and adults (Herry et al. 2006; Kim et al. 2009) but also infant animals (Kim et al. 2009). These findings are generally consistent with studies mapping the immediate early gene c-Fos as a marker of neuronal activity in prefrontallimbic circuits in other rodent models of impaired extinction. Such studies demonstrate dampened IL and BLA activity, and patterns of increased CeA activation after extinction compared with extinguishing control animals (Muigg et al. 2008). A key result of our study was that the JuvCond-AdolesExt animals (i.e., those that had good extinction retention) unexpectedly also failed to show an extinction-induced increase in pMAPK expression in the MPFC and BLA. It was surprising that adolescent animals, regardless of when they learned fear, showed an extinction pattern of dampened mPFC and BLA MAPK activation, and high CeA activation. These findings demonstrate that even under conditions that promote good extinction, the neural correlates of extinction in adolescence are different from those recruited in animals of other ages.

In this study, neural differences between the adolescent groups showing impaired or successful extinction retention were only observed in the PVT. Inactivation or lesions of the posterior PVT after conditioning have no effect on fear extinction retention in adult animals (Padilla-Coreano et al. 2012; Li et al. 2014), but reduce freezing and conditioned suppression to a CS (Padilla-Coreano et al. 2012; Li et al. 2014), and modulate CeA activity (Padilla-Coreano et al. 2012), implicating this region in fear expression and/or the retrieval of long-term fear memories. A recent study found that activation of PVT neurons during fear re- trieval involves inputs from the PL and outputs to the CeA and that these projections maintain fear the next day (DoMonte et al. 2015). The posterior PVT has robust projections to the CeA, BLA, and bed nucleus of the stria terminalis (Li and Kirouac 2008; Hsu et al. 2014), making it well-placed to influence fear expression. The most prominent projection originating from the posterior PVT is to the lateral division of the CeA (CeL) (Li and Kirouac 2008; Vertes and Hoover 2008) and selective suppression of CeL-projecting PVT neurons impaired fear retrieval (Do-Monte et al. 2015; Penzo et al. 2015) and retrieval remained impaired the day after the silencing of these neurons (Do-Monte et al. 2015). The PVT is necessary for CS-elicited fear when animals are tested in either the same (Padilla-Coreano et al. 2012; Li et al. 2014; Do-Monte et al. 2015) or a different (Penzo et al. 2015) context as conditioning, and PVT neurons are activated when rats are returned to a context previously associated with footshock (Beck and Fibiger 1995; Yasoshima et al. 2007). In addition, PVT lesions prevent ABA renewal of extinguished reward seeking (Hamlin et al. 2009), suggesting that this region contributes to the context-induced relapse of extinguished behavior. In the present study, a marker of neuronal synaptic plasticity in the PVT was elevated in adolescents during extinction in a novel context $24 \mathrm{~h}$ after conditioning, but not when fear was learned as a juvenile, or when animals were exposed to this novel context without the CS (no extinction). Activation of MAPK signaling pathways in the posterior PVT during extinction training may alter extinction learning and/or consolidation, and promote the maintenance of fear after extinction in adolescents. Future studies could test whether posterior PVT activation during extinction training modulates neuronal activity in the amygdala and if lesions or inactivation of the PVT during extinction improve extinction retention in AdolesCond-Ext animals. Moreover, manipulations that either improve extinction retention, such as D-cycloserine (McCallum et al. 2010), or reduce renewal of extinguished fear, such as retrieval-extinction procedures (Baker et al. 2013), might reduce posterior PVT MAPK activation in adolescents.

The results of our behavioral studies show that impaired fear extinction is only observed when conditioning and extinction both occur in adolescence. It is unlikely that in the JuvCond-AdolesExt animals the strength of the fear memory decayed over time (i.e., 11-12 d) and led to improved fear extinction because delaying extinction by a longer interval of $2 \mathrm{wk}$ in adolescence did not improve extinction retention, suggesting that both recent and remote fear memories are resistant to extinction in adolescence. The present study also provides novel findings that the vulnerability to fear relapse after extinction observed in earlymid adolescence $(\sim$ P35) extends to late adolescence $(\sim \mathrm{P} 47)$. Extinction retention only improved once extinction was delayed until animals transitioned out of adolescence.

Adolescence is a time when individuals leave the safety of their family environment to explore their wider environment for reproductive success. Further, individuals must acquire skills for independence over the course of the developmental transition between youth and adulthood (Spear 2000). During this period of novelty-seeking and exploration, cues signaling danger are highly relevant, are more likely to be learned (Den and Richardson 2013), and are resistant to extinction during adolescence compared with adulthood. These transient behavioral features may reflect 
ontogenetic adaptations that are suitable at a specific developmental stage (Oppenheim 1980; Spear 2000), and may arise from a combination of pronounced brain maturation and cognitive changes during adolescence (Baker et al. 2014). In particular, the significant structural and functional maturation (e.g., synaptic pruning and changes in functional connectivity) of the PFC and amygdala during adolescence likely contributes to the alterations to fear regulation in this sensitive period.

The lack of prefrontal-amygdala plasticity-related MAPK expression in adolescent rodents is consistent with human neuroimaging studies examining the role of ventromedial PFC (vmPFC), a putative homolog of the $\mathrm{IL}$, in fear extinction in adolescents. In adults, the vmPFC is activated during extinction learning and stronger activation of this region during extinction recall is associated with improved extinction retention (Phelps et al. 2004; Milad et al. 2007). However, adolescents do not show significant PFC-amygdala interactions during fear learning and extinction (Tzschoppe et al. 2014), suggesting a cross-species lack of vmPFC-amygdala connectivity during extinction in adolescence. Further, in clinical populations, adolescents with anxiety disorders (generalized anxiety disorder and/or social phobia) show decreased resting-state vmPFC-amygdala functional connectivity (Roy et al. 2013; Hamm et al. 2014) and youth with posttraumatic stress disorder (PTSD) have reduced vmPFC gray matter volume (Keding and Herringa 2015), which was linked to reexperiencing symptoms. It therefore appears that structural and functional changes in the adolescent vmPFC and amygdala in both rodents and humans are related to altered fear extinction, anxiety, and re-experiencing symptoms of PTSD.

Exploring why adolescents exhibit a striking deficit in inhibiting fear acquired during, but not before, adolescence will likely provide novel insights that can be translated into how to individually tailor treatments to improve fear inhibition in adolescents. Given the high prevalence of anxiety disorders during adolescence (Kessler et al. 2012), such an approach will be fundamental for developing early and effective interventions for anxiety. The current findings emphasize that adolescence is a vulnerable period in which fear acquired during adolescence is difficult to inhibit at this age. Importantly, adolescents show pronounced differences in the activation of the MAPK signaling pathway during extinction not only in the mPFC, but also the amygdala and PVT. Further, this study highlights that even under conditions that promote good extinction, the neural correlates of extinction (as measured by pMAPK) in adolescence are different to those recruited in animals of other ages.

\section{Materials and Methods}

\section{Subjects}

Subjects were experimentally naive male Sprague-Dawley rats $(n=170)$ obtained from the breeding colony maintained by the School of Psychology at UNSW Australia. Rats were weaned at P21-24 and were housed in groups in plastic boxes with food and water available ad libitum. No more than one rat per litter was used per group. The colony was maintained on a $12 \mathrm{~h}$ light-dark cycle (lights on at 0700). All procedures were approved by the Animal Care and Ethics Committee at UNSW and conducted in accordance with the Australian Code of Practice for the Care and Use of Animals for Scientific Purposes (7th Edition, 2004).

\section{Apparatus}

The apparatus were similar to those used in our previous studies (see Supplemental Materials and Methods; Baker et al. 2013). In brief, fear acquisition occurred in one set of chambers (Context A) while extinction and test were conducted in a second set of chambers (Context B), which had different visual features, floor- ing, size, and lighting to Context A. The CS was a 10-sec white noise that increased noise levels by $8 \mathrm{~dB}$ above background. The US was a scrambled $0.4 \mathrm{~mA}, 1$-sec footshock.

\section{Procedure}

The behavioral procedures were based upon those previously used in our laboratory for studies on extinction in adolescent rats (Baker et al. 2013; McCallum et al. 2010). Each session began with a 2-min adaptation period (see Supplemental Table S1). Freezing, defined as the absence of all movement other than that required for respiration, was measured as the conditioned fear response (see Supplemental Materials and Methods).

\section{Conditioning}

Rats received three CS trials coterminating with the US in Context A.

\section{Extinction}

Extinction occurred in Context B and consisted of 30 nonreinforced presentations of the CS (10 s each; $10 \mathrm{~s} \mathrm{ITI).} \mathrm{For} \mathrm{statistical}$ analyses of within-session extinction, six extinction trials were averaged to represent one block of extinction. In Experiment 5, a "No Extinction" control group received conditioning and was then exposed to the extinction context without any CS presentations.

\section{Test}

Animals were tested the day after extinction in Context B, which involved a single 2-min CS.

\section{Tissue processing, immunohistochemistry, and neuronal counting}

Rats were sacrificed $1 \mathrm{~h}$ following the extinction/context-only exposure session in Experiment 5 and immunohistochemical techniques were used to measure phospho-p44-42MAPK-immunoreactivity in the MPFC, amygdala, PVT, and lateral habenula using methods previously established (see Supplemental Materials and Methods; Kim et al. 2011). Manual blind counts of pMAPK-IR neurons were conducted through the rostro-caudal extent of each brain region using a light microscope (see Supplemental Materials and Methods).

\section{Acknowledgments}

This research was supported by grants from the Australian Research Council (DP120104925) and the National Health and Medical Research Council (APP1031688) to R.R., and a UNSW Australia Science Faculty Research Grant to K.D.B., who is a National Health and Medical Research Council Peter Doherty Early Career Fellow (APP1054642). We thank Arielle R. Gray for assisting in collecting the data for Experiments 3 and 5, Gavan McNally for helpful discussions, and Metaxia Kokkinos and Dana Leidl for cross-scoring behavioral data.

\section{References}

Baker KD, McNally GP, Richardson R. 2013. Memory retrieval before or after extinction reduces recovery of fear in adolescent rats. Learn Mem 20: $467-473$.

Baker KD, Den ML, Graham BM, Richardson R. 2014. A window of vulnerability: impaired fear extinction in adolescence. Neurobiol Learn Mem 113: $90-100$.

Beck CH, Fibiger HC. 1995. Conditioned fear-induced changes in behavior and in the expression of the immediate early gene c-fos: with and without diazepam pretreatment. J Neurosci 15: 709-720.

Burgos-Robles A, Vidal-Gonzalez I, Santini E, Quirk GJ. 2007. Consolidation of fear extinction requires NMDA receptor-dependent bursting in the ventromedial prefrontal cortex. Neuron 53: 871-880. 
Casey BJ, Glatt CE, Lee FS. 2015. Treating the developing versus developed brain: translating preclinical mouse and human studies. Neuron 86: $1358-1368$

Den ML, Richardson R. 2013. Enhanced sensitivity to learning fearful associations during adolescence. Neurobiol Learn Mem 104: 92-102.

Do-Monte FH, Quiñones-Laracuente K, Quirk GJ. 2015. A temporal shift in the circuits mediating retrieval of fear memory. Nature 519: 460-463.

English J, Sweatt J. 1997. A requirement for the mitogen-activated protein kinase cascade in hippocampal long term potentiation. J Biol Chem 272: $19103-19106$

Fischer A, Radulovic M, Schrick C, Sananbenesi F, GodovacZimmermann J, Radulovic J. 2007. Hippocampal Mek/Erk signaling mediates extinction of contextual freezing behavior. Neurobiol Learn Mem 87: 149-158.

Graham BM, Milad MR. 2011. The study of fear extinction: implications for anxiety disorders. Am J Psychiatry 168: 1255-1265.

Hamlin AS, Clemens KJ, Choi EA, McNally GP. 2009. Paraventricular thalamus mediates context-induced reinstatement (renewal) of extinguished reward seeking. Eur J Neurosci 29: 802-812.

Hamm LL, Jacobs RH, Johnson MW, Fitzgerald DA, Fitzgerald KD, Langenecker SA, Monk CS, Phan KL. 2014. Aberrant amygdala functional connectivity at rest in pediatric anxiety disorders. Biol Mood Anxiety Disord 4: 15.

Herry C, Trifilieff P, Micheau J, Lüthi A, Mons N. 2006. Extinction of auditory fear conditioning requires MAPK/ERK activation in the basolateral amygdala. Eur J Neurosci 24: 261-269.

Herry C, Ciocchi S, Senn V, Demmou L, Müller C, Lüthi A. 2008. Switching on and off fear by distinct neuronal circuits. Nature 454: 600-606.

Hsu DT, Kirouac GJ, Zubieta J-K, Bhatnagar S. 2014. Contributions of the paraventricular thalamic nucleus in the regulation of stress, motivation, and mood. Front Behav Neurosci 8: 73.

Huang Y-Y, Martin KC, Kandel ER. 2000. Both protein kinase A and mitogen-activated protein kinase are required in the amygdala for the macromolecular synthesis-dependent late phase of long-term potentiation. J Neurosci 20: 6317-6325.

Keding TJ, Herringa RJ. 2015. Abnormal structure of fear circuitry in pediatric post-traumatic stress disorder. Neuropsychopharmacology 40: $537-545$.

Kessler RC, Avenevoli S, Costello EJ, Georgiades K, Green JG, Gruber MJ, He J-P, Koretz D, McLaughlin KA, Petukhova M, et al. 2012. Prevalence, persistence, and sociodemographic correlates of DSM-IV disorders in the National Comorbidity Survey Replication Adolescent Supplement. Arch Gen Psychiatry 69: 372-380.

Kim JH, Hamlin AS, Richardson R. 2009. Fear extinction across development: the involvement of the medial prefrontal cortex as assessed by temporary inactivation and immunohistochemistry. J Neurosci 29: 10802-10808.

Kim SC, Jo YS, Kim IH, Kim H, Choi J-S. 2010. Lack of medial prefrontal cortex activation underlies the immediate extinction deficit. J Neurosci 30: $832-837$.

Kim JH, Li S, Richardson R. 2011. Immunohistochemical analyses of long-term extinction of conditioned fear in adolescent rats. Cereb Cortex 21: 530-538.

Kim-Cohen J, Caspi A, Moffitt TE, Harrington H, Milne BJ, Poulton R. 2003. Prior juvenile diagnoses in adults with mental disorder: developmental follow-back of a prospective-longitudinal cohort. Arch Gen Psychiatry 60: $709-717$.

Knapska E, Macias M, Mikosz M, Nowak A, Owczarek D, Wawrzyniak M, Pieprzyk M, Cymerman IA, Werka T, Sheng M, et al. 2012. Functional anatomy of neural circuits regulating fear and extinction. Proc Natl Acad Sci 109: 17093-17098.

Li S, Kirouac GJ. 2008. Projections from the paraventricular nucleus of the thalamus to the forebrain, with special emphasis on the extended amygdala. J Comp Neurol 506: 263-287.

Li Y, Dong X, Li S, Kirouac GJ. 2014. Lesions of the posterior paraventricular nucleus of the thalamus attenuate fear expression. Front Behav Neurosci 8: 94 .

Matsumoto M, Hikosaka O. 2009. Representation of negative motivational value in the primate lateral habenula. Nat Neurosci 12: 77-84.

McCallum J, Kim JH, Richardson R. 2010. Impaired extinction retention in adolescent rats: effects of D-cycloserine. Neuropsychopharmacology 35: $2134-2142$.
Milad MR, Quirk GJ. 2002. Neurons in medial prefrontal cortex signal memory for fear extinction. Nature 420: 70-74.

Milad MR, Wright CI, Orr SP, Pitman RK, Quirk GJ, Rauch SL. 2007. Recall of fear extinction in humans activates the ventromedial prefrontal cortex and hippocampus in concert. Biol Psychiatry 62: 446-454.

Morgan MA, Romanski LM, LeDoux JE. 1993. Extinction of emotional learning: contribution of medial prefrontal cortex. Neurosci Lett 163: 109-113.

Muigg P, Hetzenauer A, Hauer G, Hauschild M, Gaburro S, Frank E, Landgraf R, Singewald N. 2008. Impaired extinction of learned fear in rats selectively bred for high anxiety-evidence of altered neuronal processing in prefrontal-amygdala pathways. Eur J Neurosci 28: $2299-2309$.

Oppenheim RW. 1980. Metamorphosis and adaptation in the behavior of developing organisms. Dev Psychobiol 13: 353-356.

Orsini CA, Maren S. 2012. Neural and cellular mechanisms of fear and extinction memory formation. Neurosci Biobehav Rev 36: 1773-1802.

Padilla-Coreano N, Do-Monte FH, Quirk GJ. 2012. A time-dependent role of midline thalamic nuclei in the retrieval of fear memory. Neuropharmacology 62: 457-463.

Park J, Choi JS. 2010. Long-term synaptic changes in two input pathways into the lateral nucleus of the amygdala underlie fear extinction. Learn Mem 17: 23-34.

Pattwell SS, Duhoux S, Hartley CA, Johnson DC, Jing D, Elliott MD, Ruberry EJ, Powers A, Mehta N, Yang RR, et al. 2012. Altered fear learning across development in both mouse and human. Proc Natl Acad Sci 109: 16318-16323.

Penzo MA, Robert V, Tucciarone J, De Bundel D, Wang M, Van Aelst L, Darvas M, Parada LF, Palmiter RD, He M, et al. 2015. The paraventricular thalamus controls a central amygdala fear circuit. Nature 519: 455-459.

Phelps EA, Delgado MR, Nearing KI, LeDoux JE. 2004. Extinction learning in humans: role of the amygdala and vmPFC. Neuron 43: 897-905.

Ressler KJ, Paschall G, Zhou XL, Davis M. 2002. Regulation of synaptic plasticity genes during consolidation of fear conditioning. J Neurosci 22: 7892-7902.

Roy AK, Fudge JL, Kelly C, Perry JSA, Daniele T, Carlisi C, Benson B, Castellanos FX, Milham MP, Pine DS, et al. 2013. Intrinsic functional connectivity of amygdala-based networks in adolescent generalized anxiety disorder. J Am Acad Child Adolesc Psychiatry 52: 290-299 e292.

Santini E, Ge H, Ren K, Pena de Ortiz S, Quirk GJ. 2004. Consolidation of fear extinction requires protein synthesis in the medial prefrontal cortex. J Neurosci 24: 5704-5710.

Sierra-Mercado D Jr, Corcoran KA, Lebrón-Milad K, Quirk GJ. 2006 Inactivation of the ventromedial prefrontal cortex reduces expression of conditioned fear and impairs subsequent recall of extinction. Eur $J$ Neurosci 24: 1751-1758.

Sotres-Bayon F, Quirk GJ. 2010. Prefrontal control of fear: more than just extinction. Curr Opin Neurobiol 20: 231-235.

Sotres-Bayon F, Bush DEA, LeDoux JE. 2007. Acquisition of fear extinction requires activation of NR2B-containing NMDA receptors in the lateral amygdala. Neuropsychopharmacology 32: 1929-1940.

Spear LP. 2000. The adolescent brain and age-related behavioral manifestations. Neurosci Biobehav Rev 24: 417-463.

Sweatt JD. 2004. Mitogen-activated protein kinases in synaptic plasticity and memory. Curr Opin Neurobiol 14: 311-317.

Tzschoppe J, Nees F, Banaschewski T, Barker GJ, Buchel C, Conrod PJ, Garavan H, Heinz A, Loth E, Mann K, et al. 2014. Aversive learning in adolescents: modulation by amygdala-prefrontal and amygdala-hippocampal connectivity and neuroticism. Neuropsychopharmacology 39: 875-884.

Vertes RP, Hoover WB. 2008. Projections of the paraventricular and paratenial nuclei of the dorsal midline thalamus in the rat. J Comp Neurol 508: 212-237.

Yasoshima Y, Scott TR, Yamamoto T. 2007. Differential activation of anterior and midline thalamic nuclei following retrieval of aversively motivated learning tasks. Neuroscience 146: 922-930.

Received July 2, 2015; accepted in revised form August 21, 2015. 


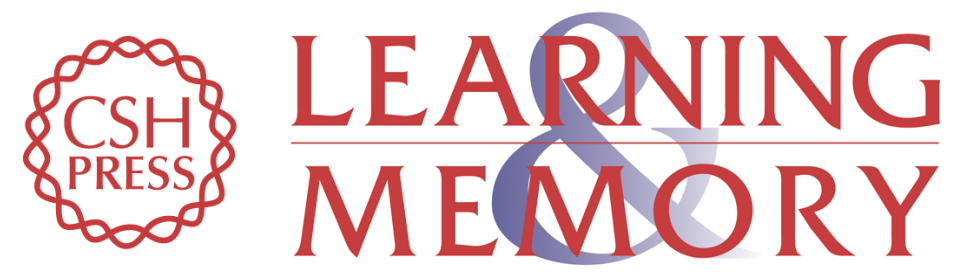

\section{Forming competing fear learning and extinction memories in adolescence makes fear difficult to inhibit}

Kathryn D. Baker and Rick Richardson

Learn. Mem. 2015, 22:

Access the most recent version at doi:10.1101/lm.039487.114

\section{Supplemental http://learnmem.cshlp.org/content/suppl/2015/10/13/22.11.537.DC1 Material}

References This article cites 49 articles, 11 of which can be accessed free at: http://learnmem.cshlp.org/content/22/11/537.full.html\#ref-list-1

Creative This article is distributed exclusively by Cold Spring Harbor Laboratory Press for the Commons first 12 months after the full-issue publication date (see

License http://learnmem.cshlp.org/site/misc/terms.xhtml). After 12 months, it is available under a Creative Commons License (Attribution-NonCommercial 4.0 International), as described at http://creativecommons.org/licenses/by-nc/4.0/.

Email Alerting Receive free email alerts when new articles cite this article - sign up in the box at the Service top right corner of the article or click here. 